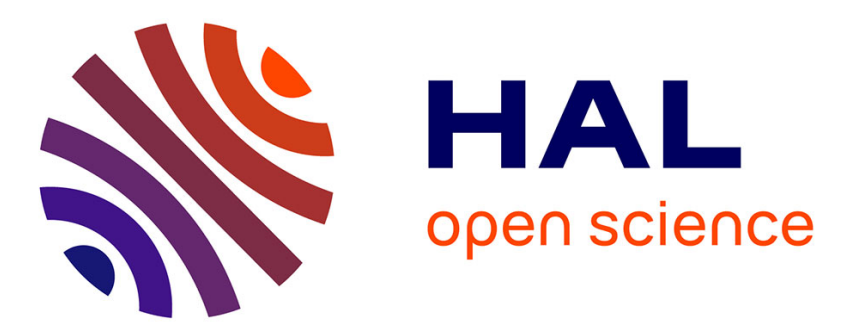

\title{
Quantification of microporosity in fruit by MRI at various magnetic fields: Comparison with X-ray microtomography
}

M. Musse, F. de Guio, S. Quellec, M. Cambert, S. Challois, A. Davenel

\section{To cite this version:}

M. Musse, F. de Guio, S. Quellec, M. Cambert, S. Challois, et al.. Quantification of microporosity in fruit by MRI at various magnetic fields: Comparison with X-ray microtomography. Journal of Magnetic Resonance Imaging, 2010, 28 (10), p. 1525 - p. 1534. 10.1016/j.mri.2010.06.028 . hal00788585

\section{HAL Id: hal-00788585 \\ https://hal.science/hal-00788585}

Submitted on 14 Feb 2013

HAL is a multi-disciplinary open access archive for the deposit and dissemination of scientific research documents, whether they are published or not. The documents may come from teaching and research institutions in France or abroad, or from public or private research centers.
L'archive ouverte pluridisciplinaire HAL, est destinée au dépôt et à la diffusion de documents scientifiques de niveau recherche, publiés ou non, émanant des établissements d'enseignement et de recherche français ou étrangers, des laboratoires publics ou privés. 


\section{Quantification of microporosity in fruit by MRI at various magnetic fields: Comparison with X-ray microtomography}

Maja Musse*, François De Guio, Stéphane Quellec, Mireille Cambert, Sylvain Challois, Armel Davenel

Cemagref, UR TERE, 17 avenue de Cucillé, CS 64427, F-35044 Rennes France.

Université européenne de Bretagne, France.

* Corresponding author

Tel: +332234821 79; Fax: +33223482115

E-mail address: maja.musse@cemagref.fr

\section{ABSTRACT}

Microstructure determines the mechanical and transport properties of fruit tissues. One important characteristic of the microstructure is the relative volume fraction of gas-filled intercellular spaces, i. e. the tissue microporosity. Quantification of this microporosity is fundamental for investigating the relationship between gas transfer and various disorders in fruit.

We present a new method for quantifying the apparent microporosity using Magnetic Resonance Imaging (MRI). The method is based on the differences in magnetic susceptibility between gas-filled intercellular spaces and their environment inside fruit tissues. This was tested at two different magnetic fields $(1.5 \mathrm{~T}$ and $0.2 \mathrm{~T})$ on apple and tomato fruit. The method was validated by comparing the MRI results with estimation of local tissue porosity using X-ray microtomography experiments. MRI was shown to be effective in determining the distribution of apparent microporosity in fruit.

Keywords: MRI; Microporosity; X-ray; Magnetic susceptibility; Plant; 


\section{INTRODUCTION}

Gas exchange between plants and their environment is essential for metabolic processes such as photosynthesis and respiration [1]. The gas-filled intercellular spaces are considered to be the predominant pathways for gas transport through a plant and are important to the characteristics of gas exchange [2]. Understanding of intercellular air spaces in relation to microstructural properties thus has important applications to understanding of several phenomena such as postharvest quality or shelf life of the fruit [3]. For example, apple fruit with greater fractional air volumes have been shown to be softer [4] or more mealy [5] and to have higher internal gas diffusion rates [6]. The volume of intercellular air spaces continues to increase during apple storage. Its measurement can therefore be used to define the age of a fruit and also to characterize the effects of different storage conditions on its quality $[5,7]$. However, the role of gas transfer in the occurrence of disorders is still not fully understood, one of the main reasons being that quantification of the intercellular air spaces is difficult.

Recent studies have used three-dimensional X-ray computed micro- and synchrotron tomography to characterize the structural arrangement of cells and voids in apple tissues $[1,3]$. The technique allows analysis of the architecture with a resolution as high as a few micrometers. Individual cell morphology, cell walls and entire void networks can thus be visualised and microporosity of tissues can be estimated via image processing methods. The main drawback of this method is the small field of view of X-ray images (in the order of 1 $\mathrm{mm}^{2}$ ). Information is consequently very local and insufficient to describe entire fruit with heterogeneous structures. Moreover, as the method requires taking samples from the fruit, it is not possible to perform continuous study on the same fruit.

Magnetic Resonance Imaging (MRI) is an appropriate tool to study plants [8] as it is noninvasive (essential to monitor dynamic processes such as growth or maturation) and has a good trade-off between spatial and temporal resolution. It can be a source of quantitative data useful to assess various mechanisms of interest because relaxation times and proton density are known to be related to the microstructure of plant tissues $[9,10]$. The presence of 
air bubbles in tomato tissues has recently been detected via gradient-echo MRI images $[11,12]$ using the effects of differences in magnetic susceptibility $(\Delta x)$ between gas bubbles and their environment inside fruit tissues. McCarthy et al [13] used susceptibility effects to study bruising in apple tissue. Duce et al. [14] investigated courgette tissues and HernandezSanchez et al. studied pears [15] using the same phenomena. MRI methods taking advantage of susceptibility effects have also several different applications such as the tracking or quantification of superparamagnetic particles [16], the detection of muscle tissue [17] and the estimation of bubble size in bread [18]. However, no MRI methods have been reported to quantify air volume, i.e. porosity, in fruit. We therefore propose here an original MRI method for quantifying porosity in intact fruit. The method was tested at two different magnetic fields on apples and tomatoes. These fruit were taken as models as they exhibit different internal structures and several ranges of porosity. The method was validated by comparing the MRI results with estimation of local tissue porosity using X-ray microtomography experiments.

\section{THEORETICAL ASPECTS}

Differences in magnetic susceptibility between a tissue and air create field inhomogeneities which induce intravoxel dephasing and associated signal loss in gradientecho (GE) images. Because of the rephasing m-pulse, signal loss occurring in spin-echo (SE) images is drastically lower and can serve as a reference. The local field inhomogeneities can be divided into three categories: macroscopic, mesoscopic and microscopic. Macroscopic inhomogeneities arise from imperfections in the magnet or large air inclusions which can lead to a non-exponential GE signal decay [19]. In the absence of macroscopic field inhomogeneities, the signal decay in GE imaging can be estimated by an exponential function described by the $\mathrm{T}_{2}{ }^{*}$ constant. Microscopic field inhomogeneities (magnetic field inhomogeneities over distances with orders of magnitude much smaller than the voxel size, i.e. atomic or molecular size) are responsible for irreversible dephasing, namely $T_{2}$ relaxation $\left(R_{2}=1 / T_{2}\right)$, and mesoscopic field inhomogeneities (from perturbers smaller than the voxel 
size but greater than the diffusion length) contribute to $R_{2}{ }^{\#}$, the reversible part of $R_{2}{ }^{*}\left(R_{2}{ }^{*}=\right.$ $\left.R_{2}+R_{2}^{\#}=1 / T_{2}{ }^{*}\right)$ [20-23]. The latter are of special interest as they originate from internal, tissue-specific sources and thus can provide important information on biological microstructure.

Using the static dephasing regime $[20,23]$ for spherical and randomly distributed perturbers in a homogenous medium, $\mathrm{R}_{2}^{\#}$ can be approximated using Eq. (1):

$$
\mathrm{R}_{2}^{\#}=\frac{2 \pi}{9 \sqrt{3}} \varepsilon \gamma \Delta \chi \mathrm{B}_{0}
$$

where $\varepsilon$ is the microporosity, $\gamma$ the proton gyromagnetic ratio $\left(\gamma=2.675^{\times} 10^{8} \mathrm{rad} / \mathrm{sT}\right.$ for the hydrogen nucleus), $\Delta \mathrm{x}$ corresponds to the difference in magnetic susceptibility between perturbers and their environment and $B_{0}$ is the main magnetic field value. The static dephasing approach has been used as a means of calculating the $T_{2}{ }^{*}$ in parenchymal lung tissue function of the alveoli volume fraction $[23,24]$ and to assess the density of iron-oxide nanoparticles [25].

In the study presented here, the microporosity was the gas volume fraction due to intercellular spaces in the fruit tissues. $\Delta \mathrm{X}$ was taken as $-9 \mathrm{ppm}$ to represent air/water susceptibility interfaces. According to [26], if a mesoscopic inhomogeneous magnetic field is created by spherical objects with an average radius of $R_{0}$, the static dephasing regime holds if the characteristic time $t_{C}$ is much smaller than the characteristic diffusion time $t_{D}\left(t_{D}=R_{0}{ }^{2} / D\right)$ :

$$
\mathrm{t}_{\mathrm{C}}=9 \sqrt{3} /\left(2 \pi \gamma \Delta \chi \mathrm{B}_{0}\right)<<\mathrm{t}_{\mathrm{D}}
$$

where $D$ is the diffusion coefficient. Eq. (2) shows that a high external magnetic field $\left(\mathrm{B}_{0}\right)$, wide susceptibility differences $(\Delta \chi)$ and large susceptibility inclusions favour the static dephasing regime. For a given $\mathrm{B}_{0}$ and $\Delta \chi$, conditions for which the static dephasing regime is valid are met for the radii given by Eq. 3 :

$$
R_{0}^{2}>>\frac{9 \sqrt{3} D}{2 \pi \gamma \Delta \chi B_{0}}
$$

The estimation of porosity from Eq. (1) is straightforward and given in Eq. (4): 
$\varepsilon=\frac{9 \sqrt{3} R_{2}^{\#}}{2 \pi \gamma \Delta \chi B_{0}}$

$\mathrm{R}_{2}{ }^{\#}$ can be estimated according to the following equation (Eq. (5)):

$\mathrm{R}_{2}^{\#}=\mathrm{R}_{2}^{*}-\mathrm{R}_{2}$

Finally, by combining multiple spin-echo images to assess $R_{2}$ and multiple gradient-echo images to assess $\mathrm{R}_{2}{ }^{*}$, we were able to estimate microporosity in each fruit. As computation of microporosity is dependent on $\mathrm{B}_{0}$, as can be seen in Eq. (4), the method was tested at 1.5 and $0.2 \mathrm{~T}$.

\section{MATERIALS AND METHODS}

\subsection{Plant material}

Nine Royal gala apples were procured from the local market and used without any processing. Fruit were harvested in October 2008 and stored by the producer until July 2009 when the experiment was performed.

Eight Admiro tomatoes provided by the Ctifl (Centre Technique Interprofessionel des Fruits et Légumes, France) were used in this study. Fruit were collected in April 2009 at the late green stage (tomato colour code 3-4, Ctifl, France) and left to ripen in a constantly aerated ripening chamber under controlled conditions $\left(18^{\circ} \mathrm{C}\right.$ and $\left.55 \% \mathrm{RH}\right)$. Three tomatoes were studied the first day, three tomatoes the second day and two tomatoes the third day, the ripening period was thus eight, nine and ten days, for the first, second and third groups, respectively.

\subsection{Magnetic Resonance Imaging}

\subsubsection{Image acquisition}

Measurements were carried out on a $1.5 \mathrm{~T}$ scanner (Magnetom Avanto, Siemens, Erlangen, Germany) with the maximum imaging gradients of $45 \mathrm{mT} / \mathrm{m}$ for the longitudinal (z) and $40 \mathrm{mT} / \mathrm{m}$ for the transverse ( $\mathrm{x}$ and $\mathrm{y}$ ) axes, and on a $0.2 \mathrm{~T}$ scanner (Magnetom Open, 
Siemens, Erlangen, Germany) with the maximum imaging gradients of $15 \mathrm{mT} / \mathrm{m}$ along all axes. The 1.5 $\mathrm{T}$ and $0.2 \mathrm{~T}$ scanners were equipped with the 8-channel "knee" receiver coil and multipurpose flexible receiver coil (MP_S), respectively. Fruit were positioned in a specifically designed experimental support and marked before the first experiment making it possible to position them very close to the same position in both scanners. In order to guarantee a constant temperature for the fruit during measurements, they were placed in Faraday cages at least one day before the experiment began. The temperature of the thermally insulated Faraday cages was set at $21^{\circ} \mathrm{C}$ and monitored during measurements (deviation was less than $0.5^{\circ} \mathrm{C}$ ).

The median equatorial plane of each fruit was imaged with the following geometrical parameters: matrix size $=128^{2}$ pixels, field of view $=128^{2} \mathrm{~mm}^{2}$ and slice thickness $=5 \mathrm{~mm}$. The repetition time (TR) was set at $10 \mathrm{~s}$ for all measurements.

For the tomatoes, $T_{2}$ relaxation maps were obtained from a multi-spin echo sequence (MSE) with 32 consecutive echoes (first echo equal to inter-echo spacing), ranging from 15 to $480 \mathrm{~ms}$ and from 30 to $960 \mathrm{~ms}$ at $1.5 \mathrm{~T}$ and $0.2 \mathrm{~T}$, respectively. The MRI signal was accumulated twice for measurements at $0.2 \mathrm{~T} \mathrm{~T}_{2}{ }^{*}$ relaxation maps were obtained at $1.5 \mathrm{~T}$ from a multi-gradient echo sequence (MGE) with 12 consecutive equally spaced echoes ranging from 4 to $48 \mathrm{~ms}$. Six separate GE images with TE of 4, 8, 12, 16, 40 and 80 ms were acquired for $\mathrm{T}_{2}{ }^{*}$ maps at $0.2 \mathrm{~T}$ (MGE sequence was not available). The same sequences were used for the apples as for tomatoes, except that eight separate GE images with TE of $4,8,12,16,20,30,50$ and $70 \mathrm{~ms}$ were acquired for $\mathrm{T}_{2}{ }^{*}$ maps at $0.2 \mathrm{~T}$.

\subsubsection{Image processing}

The magnitudes of the MSE and MGE image series were fitted on a pixel-by-pixel basis using corresponding monoexponential functions, via the Levenberg-Marquardt criterion for chi-square minimization implemented on Scilab software. Regions of interest (ROI) were defined manually from the MSE images (Fig. 1) that corresponded to a homogeneous region in different tomato tissues (core, placenta, radial and outer pericarp and locular tissue) and in 
the intermediate region of apple cortex. As different parts of apple cortex could not be distinguished in MSE images, the ROls were drawn at about $1.5 \mathrm{~cm}$ from the skin in the intermediate cortex layer from which samples were taken for X-ray experiments. The average values of $T_{2}, T_{2}{ }^{*}$ and microporosity were measured within these regions.

\subsection{X-ray microtomography}

\subsubsection{Image acquisition}

The samples were scanned using an X-ray micro CT system (Phoenix, VTome X 240, General Electric, Wunstorf, Germany) operating at a voltage of $120 \mathrm{kV}$ and a current of 83 $\mu \mathrm{A}$. A total of 800 projections with an exposure time of $1 \mathrm{~s}$ was acquired for each sample over the interval $0-360^{\circ}$, resulting in a pixel resolution of $10^{2} \mu \mathrm{m}^{2}$. Three projections were averaged for each slice image. The tomographic reconstruction was performed using the Datos Xrec Software (Phoenix Xray). A stack of 200 2-D cross sections with a $10 \mu \mathrm{m}$ distance was then used for reconstruction of the full 3-D structure of the samples.

Samples were taken from the fleshy part (cortical tissue) of apples as follows: a 2-cm thick slice, centered over the middle of the virtual MRI slice (Fig. 2 A), was cut in the equatorial region perpendicular to the pedicle axis, and cylinders $(0.8 \mathrm{~cm}$ in diameter) were cut into the tissue at about $1.5 \mathrm{~cm}$ from the skin to the axis of the tissue cylinder (Fig. $2 \mathrm{~B}$ ). Each sample was directly positioned on a system needle. The same procedure was used for extraction of samples of the outer pericarp of tomatoes. Five apple samples and one tomato sample were studied by X-ray tomography. The measurement time for each sample was around 45 minutes.

\subsubsection{Image processing}

A circular region of interest that excluded the image edges was extracted from the images and processed., which mainly consisted of image segmentation and construction of the 3-D representation of the void network. 
Segmentation consisted of converting the grey scale image into a binary image by determining the population assignment for each voxel of the image. Assuming that the image contained two classes of pixels (e.g. cells and voids), the Otsu method [27], which calculates the optimum threshold separating these two classes, was implemented in Scilab software and used for automatic image segmentation. The reconstructed binary images, with voxels identified as either void or cell, were then used to compute the microporosity by dividing the number of voxels corresponding to the voids by the total number of voxels. For comparison with MRI results, only 200 X-ray images (that corresponded to the 5-mm sample thickness) centred over the middle of the sample were used for mean microporosity computing.

MicroView software was used for the 3-D representation of the void network.

\section{RESULTS}

\subsection{MRI estimation of microporosity at $1.5 \mathrm{~T}$ and $0.2 \mathrm{~T}$ magnetic fields}

$T_{2}$ and $T_{2}{ }^{*}$ maps of one apple, calculated from the sets of SE and GE images at multiple echo times obtained at 1.5 $\mathrm{T}$ and $0.2 \mathrm{~T}$, are shown in Fig. 3. Both $\mathrm{T}_{2}$ and $\mathrm{T}_{2}{ }^{*}$ maps reflect the heterogeneity of the apple fruit tissues. The major morphological parts of the fruit, i. e. seeds, cortex and vascular bundles, can be distinguished. $T_{2}$ and $T_{2}{ }^{*}$ maps were also computed for the tomatoes (Fig. 4) making it possible to differentiate different tissues such as the core, placenta, radial pericarp, outer pericarp and locular tissue.

The results of $T_{2}$ and $T_{2}{ }^{*}$ measurements are summarised in Table 1. A decrease in $T_{2}$ and $\mathrm{T}_{2}{ }^{*}$ relaxation times with increasing magnetic field strength can be observed both for apple and tomato tissues. The $\mathrm{T}_{2}{ }^{*}$ dependence on the magnetic field was expected as intravoxel dephasing in gradient-echo imaging scales with the main magnetic field value. On the other hand, the $T_{2}$ decrease with higher fields was a result of increased dynamic dephasing due to increased local magnetic field inhomogeneities created at the interfaces of the tissues with different magnetic susceptibilities [28]. As molecules diffuse through these microscopic field gradients, they lose phase coherence which is not refocused by m-pulse, resulting in a shorter apparent $T_{2}$. The susceptibility gradients increase linearly in strength 
with the main magnetic field, and the diffusion term responsible for dynamic dephasing scales with the second power of the gradient strength. Proton chemical exchange between water and dissolved solutes (especially sugars in ripe fruit) can be another origin of a $T_{2}$ dependence on the magnetic field strength and pulse spacing [14]. Note that the inter-echo spacing of the MSE sequence was $15 \mathrm{~ms}$ at $1.5 \mathrm{~T}$ and $30 \mathrm{~ms}$ at $0.2 \mathrm{~T}$ in order to limit the dephasing effect at $1.5 \mathrm{~T}$ and thus that the $\mathrm{T}_{2}$ values measured at two fields are not directly comparable.

The parametric $T_{2}$ and $T_{2}{ }^{*}$ images shown in Fig. 3 and Fig. 4 were used to compute porosity maps of apple and tomato fruit. An example of the porosity maps measured at $1.5 \mathrm{~T}$ is shown in Fig. 5. Like the $T_{2}$ and $T_{2}{ }^{*}$ maps, the porosity image of the apple (Fig. $5 \mathrm{~A}$ ) allowed differentiation of seeds, cortex and vascular bundles. It was clearly visible that the porosity inside the cortex tissue was not uniform. In fact, porosity decreased gradually from the cortical tissue near to seeds $(24 \pm 4 \%$ at $1.5 \mathrm{~T}$, in the example shown) to the tissue under the skin $(16 \pm 2 \%$ at $1.5 \mathrm{~T}$, in the example shown). The mean porosity value in the intermediate region was $21 \pm 2 \%$ at $1.5 \mathrm{~T}$. This intermediate region was considered in the numerical results presented later in this study.

An example of a porosity map of the tomato is depicted in Fig. 5 B showing the heterogeneity of porosity in tomatoes. The core had the largest proportion of air bubbles $(15 \pm 4 \%)$, while the locular tissue was shown to be almost gas free, with a mean porosity of less than $1 \%$. The mean porosity in the placenta, the radian and the outer pericarp was $11 \pm 2 \%, 4 \pm 1 \%$ and $1 \pm 0 \%$, respectively. These results entirely agree with the macrovision images reported in our previous study [11].

The mean porosity of the apple and tomato tissues measured at $1.5 \mathrm{~T}$ and $0.2 \mathrm{~T}$ are summarised in Table 2.

The relationship (Fig. 6) between mean porosity in different tomato tissues and the apple cortex measured at $1.5 \mathrm{~T}$ and $0.2 \mathrm{~T}$ was linear: porosity $\mathrm{1}_{1.5 \mathrm{~T}}=1.4$ porosity $\mathrm{y}_{0.2}-0.7$, with $\mathrm{r}^{2}=0.97$, 
showing that the results matched well. However, the slope of the curve indicated that the porosity values measured at $1.5 \mathrm{~T}$ were greater than values obtained at $0.2 \mathrm{~T}$.

\subsection{X-ray microtomography images}

An example of the 2-D X-ray tomographic slices of the apple cortex is shown in Fig. 7, with ROI determining pixels used for estimation of microporosity. Dark regions on the image correspond to gas-filled intercellular spaces and light regions correspond to cells. The contrast between these two tissue types is well differentiated and the spatial resolution of the images is sufficient for estimation of porosity. As observed by Verboven et al [1], voids in the apple often appeared larger than surrounding cells and some cells were not connected to an intercellular space.

The quality of the tomato X-ray images was very poor and not exploitable for estimation of porosity. This was explained by the very high water content of the samples and their significant deterioration during the 45 minutes' measurement time. No improvement was observed when samples were enclosed in plastic film.

Fig. 8 shows a 3-D representation of the pore structure of the cylindrical apple sample. This simple volume representation provides better understanding of the internal tissue organisation and complexity of the cell and void distribution.

\subsection{Comparison of the MRI and X-ray microtomography estimations of porosity}

The results of the MRI porosity measurements in the apple cortex tissue were compared to the porosity estimated from the X-ray tomography experiments (Fig. 9). Five apples were studied by both MRI at $1.5 \mathrm{~T}$ and X-ray microtomography. The relationship between the measurements was linear (porosity $\mathrm{IRM}_{\mathrm{IR}(1.5 \mathrm{~T})}=0.9$ porosity $\left.\mathrm{X}_{\mathrm{-rays}}+5\right)$, with the correlation coefficient $r^{2}=0.98$. Two of the apples were also studied by $0.2 \mathrm{~T} \mathrm{MRI}$, and the results of these measurements are presented in Fig. 9. Because of the small number of points (two), no relationship could be established between the measurements. The relatively high standard 
deviation of the mean values of the MRI porosity measurements represented on the graph corresponds to natural structural variation in the tissue.

\section{DISCUSSION}

The results from the experimental study at $1.5 \mathrm{~T}$ and $0.2 \mathrm{~T}$ were in good agreement. The relationship obtained between the two measurements was linear with an offset of nearly zero. However, the slope of the line differed slightly from that expected $(x=y)$, possibly explained by the hypotheses assumed in the model for mesoscopic inhomogeneities described by Yablonskiy [26] or by the methods used for the relaxation time measurements.

The model for mesoscopic inhomogeneities is valid for a broad range of perturber strengths and sphere sizes as long as following conditions are satisfied : i) the magnetic perturbers are uniform spheres; ii) there are no interactions between spheres (small volume fraction of the perturber subsystem, i.e. $\varepsilon<<100 \%$ ) and iii) the system under investigation is in the static dephasing regime.

i) The intercellular spaces were described in this study as gas-filled spherical volumes of the same size. A regular spherical geometry was a first-order approximation, but it is obvious in the X-ray images that not all the spaces were spherical and that they were not uniform in size.

ii) It is assumed in the model that susceptibility inclusions such as gas-filled intercellular spaces constitute a minor subsystem in an imaging voxel and represent a low volume fraction of the whole system. The criterion of low volume fraction is approximate and the boundaries within which the theoretical approach is valid were not determined [20]. The experimental results obtained from a phantom showed that the method provided good results for measurements of porosity below $6 \%$ [26]. In a recent study on the mouse lung [24], the method was used for investigation of porosity levels between $30 \%$ and $65 \%$, but no external reference was used to validate these results. In the present study, maximum values of porosity of about $20 \%$ were measured in the apples. 
iii) Finally, the model for mesoscopic inhomogeneities operates for a static dephasing regime which holds if the characteristic time $\left(t_{c}\right)$ is much smaller that the characteristic diffusion time ( $t_{D}$ ) (as described in Eq. 2). It is obvious from Eq. 2 that the static regime is favoured by a high external magnetic field $\left(B_{0}\right)$, wide susceptibility differences $(\Delta \chi)$ and large size perturbers. Eq. 3 provides a test to determine whether the static dephasing regime is applicable to given experimental conditions. For tomato and apple studies, the susceptibility difference for the water/air interface is about $-9 \mathrm{ppm}$ and the diffusion coefficient was $1.8 \times 10^{-9}$ $\mathrm{m}^{2} / \mathrm{s}$ according to [29]. The radius of spherical perturbers has to be much greater than $1 \mu \mathrm{m}$ and $3 \mu \mathrm{m}$ for the magnetic fields of $1.5 \mathrm{~T}$ and $0.2 \mathrm{~T}$, respectively. The intercellular spaces of the apple cortex reported in the literature are considerably higher than these values. The Xray images (Fig. 7) acquired in this study also indicated that the mean radius of the intercellular spaces of the apple samples was ten to one hundred times greater than the radius limit required in the model. On the other hand, the criterion in Eq. 2 was valid only for a FID (or gradient-echo) experiment $[26,30]$ since it describes the situation when dephasing takes control of NMR signal attenuation before molecular diffusion takes over the process. For a spin-echo process, molecular diffusion is the principal mechanism that is responsible for signal loss during the echo time, due to the presence of an inhomogeneous field. Diffusion attenuation increases with the echo time [11,31] and hence imposes a restriction on the maximum echo time that can be used. If water molecules do not move too far from their original location during an interval of the echo time, i.e. spins move so slowly that they appear to be stationary, then the inhomogeneous magnetic field that the molecules experience may be well characterised by a constant local field gradient. Diffusion thus has practically no effect on the relaxation, and the loss of phase coherence is completely recovered with the refocusing $\pi$-pulse. With regard to the results of $T_{2}$ measurements (Table 1), the diffusion signal loss occurred in the spin-echo measurements. For the tissues rich in air, the $T_{2}$ was significantly greater at $0.2 \mathrm{~T}$ than at $1.5 \mathrm{~T}$. Thus, $\mathrm{T}_{2}$ values reflected not only the microscopic magnetic field inhomogeneity effects, but also incorporated the effects of the 
tissue perturbers. However, for tissues characterised by long $T_{2}$ such as vegetable tissues, the error in $T_{2}$ value does not significantly influence the porosity computed from Eq. 4. For example, a $100-\mathrm{ms}$ variation in $\mathrm{T}_{2}$ led to only $0.05 \%$ variation in microporosity.

The second reason for the slight disagreement between porosity measurements performed at $1.5 \mathrm{~T}$ and $0.2 \mathrm{~T}$ may have arisen from errors in the relaxation time measurements. The transverse relaxation in apple and tomato tissues is described by a multi-exponential model reflecting different sub-cellular water compartments $[32,11]$. The MRI relaxation maps are derived from a relatively low echo number and do not make it possible to resolve contributions from different compartments. MRI thus provides an average of the true relaxation time which depends on the parameters of the imaging sequence.

As opposed to $T_{2}$ measurements, the errors in estimation of $T_{2}{ }^{*}$ may strongly influence the computation of porosity. In order to obtain accurate values, $\mathrm{T}_{2}{ }^{*}$ was computed from multipoint decay curves, but sources of error may still have remained. The first echo time was set at $4 \mathrm{~ms}$ for both MRI systems because of technical limitations. The $\mathrm{T}_{2}{ }^{*}$ of tissues rich in air, such as the apple cortex and tomato core, were about 3 and $5 \mathrm{~ms}$, respectively. Thus the minimum echo time of $4 \mathrm{~ms}$ may be a limit for accurate estimation of $\mathrm{T}_{2}{ }^{*}$. It will be relevant to evaluate the method on a MRI scanner with a faster gradient switching allowing shorter TE. In the case of measurements at $0.2 \mathrm{~T}$, the last TE was set at 70 and $80 \mathrm{~ms}$ for the apple and tomatoes, respectively. These values were not sufficiently long to reach the baseline and may also have introduced errors in the fitting results. These issues will be improved in further applications.

In the case of the tomatoes, $\mathrm{T}_{2}{ }^{*}$ maps were sensitive to macroscopic field inhomogeneities (larger than voxel size) in addition to mesoscopic inhomogeneities. Gasfilled holes were in fact present in the locular tissue of the tomatoes, particularly tomatoes 1 and 2. Voxels corresponding to the outer pericarp and locular tissues near the macroscopic holes were therefore not considered.

The MRI and X-ray porosity measurements were in relatively good agreement. The relationship obtained between the two measurements was linear, with $r^{2}=0.98$, and the slope 
close to 1 (0.9). However, the offset was greater than expected. The explanations may lie with different phenomena. Firstly, the two methods were compared in a limited range of porosities for the apples (14-25\%), because X-ray images of the tomatoes were not exploitable for estimation of porosity. It would be interesting to extend this range and to compare the MRI and X-ray methods for lower porosity values. Therefore, other fruit models, such as the pear, which is characterised by lower porosity than apples ( $19 \%$ for apples and $8 \%$ for pears [33]), will be used in our subsequent studies. Secondly, we considered in this study that $10 \mu \mathrm{m}^{3} /$ voxel resolution of X-ray images was appropriate to estimate the "reference" porosity and thus to validate the MRI method. However, X-ray results cannot be taken as absolute. Mendoza et al. [3] showed that porosity computed from X-ray images was highly dependent on the resolution used. Moreover, although particular care was taken to make MRI and X-ray experiments comparable, it was particularly difficult to consider exactly the same locality in the apple cortex for both measurements. The porosity of the apple cortex progressively changed from the centre to the skin and this factor may have led to an incorrect interpretation of the measurements. Finally, the gas in intercellular spaces was considered to be air rather than a more complex gas because the susceptibility constant of air is known. This approximation may influence the estimation of microporosity values (see Eq. 4).

Although MRI has a much lower resolution than the size of the intercellular spaces, the method used here takes advantage of the signal loss occurring in the gradient echo process due to intravoxel dephasing. Indeed, even mesoscopic magnetic perturbers smaller than the voxel size modify the voxel signal whose mean porosity can be assessed. Moreover, the method seems to be reliable as experiments using two magnets with different magnetic field strengths provided congruent results. It is also non-invasive and non-destructive, which is of great importance in plant investigations. Finally, MRI makes it possible to investigate the distribution of porosity in an entire fruit or in a chosen virtual slice in a relatively short time interval. On the other hand, X-ray Computed Tomography remains a unique technique which provides visualisation of cell morphology and void network architecture, but with the 
constraints of long acquisition times and small samples. These two techniques can thus be considered to be complementary.

At last, considering the results of the present study it is evident that both MRI and X-ray measurements reflect apparent and not absolute values of porosity. It is thus important to underline that the MRI method presented here is relevant for comparative studies.

\section{CONCLUSION}

The results of this study showed that MRI can be used for quantification of apparent microporosity in fruit tissues. The great advantage of this technique compared to other experimental methods for accessing of microporosity is that MRI makes it possible to measure the spatial distribution of porosity. Furthermore, MRI allows estimation of apparent microporosity on intact whole fruit and thus repeated measurements can be performed on an individual sample. The method is very promising for investigation of relationships between gas transfer and various disorders in fruit during postharvest life. Application of the method will be extended to other biological tissues.

\section{REFERENCES:}

1. Verboven P, Kerckhofs G, Mebatsion HK, Ho QT, Temst K, Wevers M, Cloetens P, Nicolai BM. Three-dimensional gas exchange pathways in pome fruit characterized by synchrotron x-ray computed tomography. Plant Physiol 2008;147(2):518-527.

2. Schotsmans W, Verlinden BE, Lammertyn J, Nicolai BM. The relationship between gas transport properties and the histology of apple. J Sci Food Agr 2004;84(10):11311140.

3. Mendoza F, Verboven P, Mebatsion HK, Kerckhofs G, Wevers M, Nicolai B. Threedimensional pore space quantification of apple tissue using X-ray computed microtomography. Planta 2007;226(3):559-570.

4. Yearsley $\mathrm{CW}$, Banks $\mathrm{NH}$, Ganesh $\mathrm{S}$. Temperature effects on the internal lower oxygen limits of apple fruit. Postharvest Biol Technol 1997;11(2):73-83. 
5. Tu K, De Baerdemaeker J, Deltour R, De Barsy T. Monitoring post-harvest quality of Granny Smith apple under simulated shelf-life conditions: Destructive, non-destructive and analytical measurements. Int J Food Sci Tech 1996;31(3):267-276.

6. Ho QT, Verlinden BE, Verboven P, Vandewalle S, Nicolai BM. A permeationdiffusion-reaction model of gas transport in cellular tissue of plant materials. J Exp Bot 2006;57(15):4215-4224.

7. Harker FR, Watkins CB, Brookfield PL, Miller MJ, Reid S, Jackson PJ, Bieleski RL, Bartley T. Maturity and regional influences on watercore development and its postharvest disappearance in 'Fuji' apples. J Am Soc Hortic Sci 1999;124(2):166-172.

8. Hills BP, Clark CJ. Quality assessment of horticultural products by NMR. Annual Reports on NMR Spectroscopy 2003;50:75-120.

9. Kockenberger W. Functional Imaging of Plants by Magnetic Resonance Experiments. Trends Plant Sci 2001;6(7):286-292.

10. Van As $\mathrm{H}$. Intact plant $\mathrm{MRI}$ for the study of cell water relations, membrane permeability, cell-to-cell and long distance water transport. J Exp Bot 2007;58(4):743756.

11. Musse M, Quellec S, Devaux M-F, Cambert M, Lahaye M, Mariette F. An investigation of the structural aspects of the tomato fruit by means of quantitative nuclear magnetic resonance imaging. Magn Reson Imaging 2009;27(5):709-719.

12. Musse M, Quellec S, Cambert M, Devaux M-F, Lahaye M, Mariette F. Monitoring the postharvest ripening of tomato fruit using quantitative MRI and NMR relaxometry. Postharvest Biol Technol 2009;53(1-2):22-35.

13. McCarthy MJ, Zion B, Chen P, Ablett S, Darke AH, Lillford PJ. Diamagnetic susceptibility changes in apple tissue after bruising. J Sci Food Agr 1995;67(1):13-20.

14. Duce SL, Carpenter TA, Hall LD, Hills BP. An Investigation of the Origins of Contrast in NMR Spin Echo Images of Plant Tissue. Magn Reson Imaging 1992;10:289-297.

15. Hernández-Sánchez N, Hills BP, Barreiro P, Marigheto N. An NMR study on internal browning in pears. Postharvest Biol Technol 2007;44(3):260-270. 
16. Cunningham $\mathrm{CH}$, Arai T, Yang PC, McConnell MV, Pauly JM, Conolly SM. Positive contrast magnetic resonance imaging of cells labeled with magnetic nanoparticles. Magn Reson Med 2005;53(5):999-1005.

17. Bonny J-M, Laurent W, Renou J-P. Detection of susceptibility effects using simultaneous $\mathrm{T}^{*}$ and magnetic field mapping. Magn Reson Imaging 2000;18(9):1125-1128.

18. De Guio F, Musse M, Benoit-Cattin H, Lucas T, Davenel A. Magnetic resonance imaging method based on magnetic susceptibility effects to estimate bubble size in alveolar products: application to bread dough during proving. Magn Reson Imaging $2009 ; 27(4): 577-585$

19. De Guio F, Benoit-Cattin H, Davenel A. Signal decay due to susceptibility-induced intravoxel dephasing on multiple air-filled cylinders: MRI simulations and experiments. Magn Reson Mater Phy 2008;21(4):261-271.

20. Yablonskiy DA, Haacke EM. Theory of NMR signal behavior in magnetically inhomogeneous tissues: The static dephasing regime. Magn Reson Med $1994 ; 32(6): 749-763$.

21. Sukstanskii AL, Yablonskiy DA. Theory of FID NMR signal dephasing induced by mesoscopic magnetic field inhomogeneities in biological systems. J Magn Reson 2001;151(1):107-117.

22. Fernandez-Seara MA, Wehrli FW. Postprocessing technique to correct for background gradients in image-based R-2(*) measurements. Magn Reson Med $2000 ; 44(3): 358-366$

23. Pracht ED, Arnold JFT, Wang T, Jakob PM. Oxygen-enhanced proton imaging of the human lung using T2*. Magn Reson Med 2005;53(5):1193-1196.

24. Olsson LE, Lindahl M, Onnervik PO, Johansson LB, Palmer M, Reimer MK, Hultin L, Hockings PD. Measurement of MR signal and T2* in lung to characterize a tight skin mouse model of emphysema using single-point imaging. J Magn Reson Imaging $2007 ; 25(3): 488-494$ 
25. Bowen CV, Zhang X, Saab G, Gareau PJ, Rutt BK. Application of the static dephasing regime theory to superparamagnetic iron-oxide loaded cells. Magn Reson Med 2002;48(1):52-61.

26. Yablonskiy DA. Quantitation of intrinsic magnetic susceptibility-related effects in a tissue matrix. Phantom study. Magn Reson Med 1998;39(3):417-428.

27. Otsu N. A threshold selection method from gray-level histograms. IEEE Trans Syst Man Cybern 1979;SMC-9(1):62-66.

28. de Graaf RA, Brown PB, Mclntyre S, Nixon TW, Behar KL, Rothman DL. High magnetic field water and metabolite proton T-1 and T-2 relaxation in rat brain in vivo. Magn Reson Med 2006;56(2):386-394.

29. Duval F, Cambert M, Mariette F. NMR study of tomato pericarp tissue by spin-spin relaxation and water self-diffusion. Appl Magn Reson 2005;28(1-2):29-40.

30. Yung KT. Empirical models of transverse relaxation for spherical magnetic perturbers. Magn Reson Imaging 2003;21(5):451-463.

31. Edzes HT, van Dusschoten D, Van As H. Quantitative T2 Imaging of Plant Tissues By Means Of Multi-Echo MRI Microscopy. Magn Reson Imaging 1998;16(2):185-196.

32. Marigheto N, Venturi L, Hills BP. Two-dimensional NMR relaxation studies of apple quality. Postharvest Biol Technol 2008; 48:331-340.

33. Mebatsion HK, Verboven P, Endalew AM, Billen J, Ho QT, Nicolai BM. A novel method for 3-D microstructure modeling of pome fruit tissue using synchrotron radiation tomography images. J Food Eng 2009;93(2):141-148.

\section{FIGURE CAPTIONS:}

Table 1: Mean values of $\mathrm{T}_{2}$ and $\mathrm{T}_{2}{ }^{*}$ for the apple cortex and different tomato tissues measured at $1.5 \mathrm{~T}$ and $0.2 \mathrm{~T}$. 
Table 2: Mean values of porosity for the apple cortex and different tomato tissues measured at $0.2 \mathrm{~T}$ and $1.5 \mathrm{~T}$.

Fig. 1 : MSE image of the median equatorial plane of apple $(A)$ and tomato $(B)$ obtained with MSE sequence (TE=60 and TE=120 ms, respectively) at $1.5 \mathrm{~T}$.

Fig. 2 : Schematic representation of the experimental procedure of apple sampling for X-ray microtomography measurements. A) Extraction of a 2-cm thick slice (solid lines) centered over the middle of the virtual MRI slice (dashed line). B) Extraction of the sample $(0.8 \mathrm{~cm}$ in diameter).

Fig. $3: T_{2}(A)$ and $T_{2}{ }^{*}(B)$ maps of the median plane of apple obtained at $1.5 T \cdot T_{2}(C)$ and $\mathrm{T}_{2}{ }^{*}(\mathrm{D})$ maps of the median plane of apple obtained at $0.2 \mathrm{~T}$.

Fig. $4: \mathrm{T}_{2}(\mathrm{~A})$ and $\mathrm{T}_{2}{ }^{*}(\mathrm{~B})$ maps of the median plane of tomato fruit obtained at $1.5 \mathrm{~T} . \mathrm{T}_{2}(\mathrm{C})$ and $\mathrm{T}_{2}{ }^{*}(\mathrm{D})$ maps of the median plane of tomato obtained at $0.2 \mathrm{~T}$.

Fig. 5 : Porosity maps of the median plane of apple (A) and tomato (B) measured at $1.5 \mathrm{~T}$.

Fig. 6 : Comparison between mean porosity measured at $0.2 \mathrm{~T}$ and mean porosity measured at 1.5T for apple cortex $(\bullet)$ and tomato tissues ( o - core; $\square$ - placenta; $ж$-radial pericarp; $\diamond$ outer pericarp and $\Delta$ - locular tissue). The dashed line is the linear relationship between two measurements: porosity ${ }_{1.5 T}=1.4$ porosity ${ }_{0.2 T}-0.7$ with $r^{2}=0.98$.

Fig. 7 : X-ray image with pixel resolution of $10-\mu \mathrm{m}$ before processing. Dark regions correspond to gas-filled intercellular spaces and light regions correspond to cells. ROI subjected to processing is shown as dashed line. 
Fig. 8 : 3-D representation of pore (light) structure of the cylindrical apple sample.

Fig. 9 : Comparison of MRI porosity measurements in the apple cortex tissue performed at 1.5 T (०) and 0.2 T $(\Delta)$ with X-ray porosity measurements. The dashed line is the linear relationship between the $\mathrm{MRI}$ measurements at $1.5 \mathrm{~T}$ and the $\mathrm{X}$-ray measurements: (porosity $_{1.5 T}=0.9$ porosity $_{\mathrm{X}-\text { ray }}+5$ ) with $\mathrm{r}^{2}=0.98$. 
Fig. 1
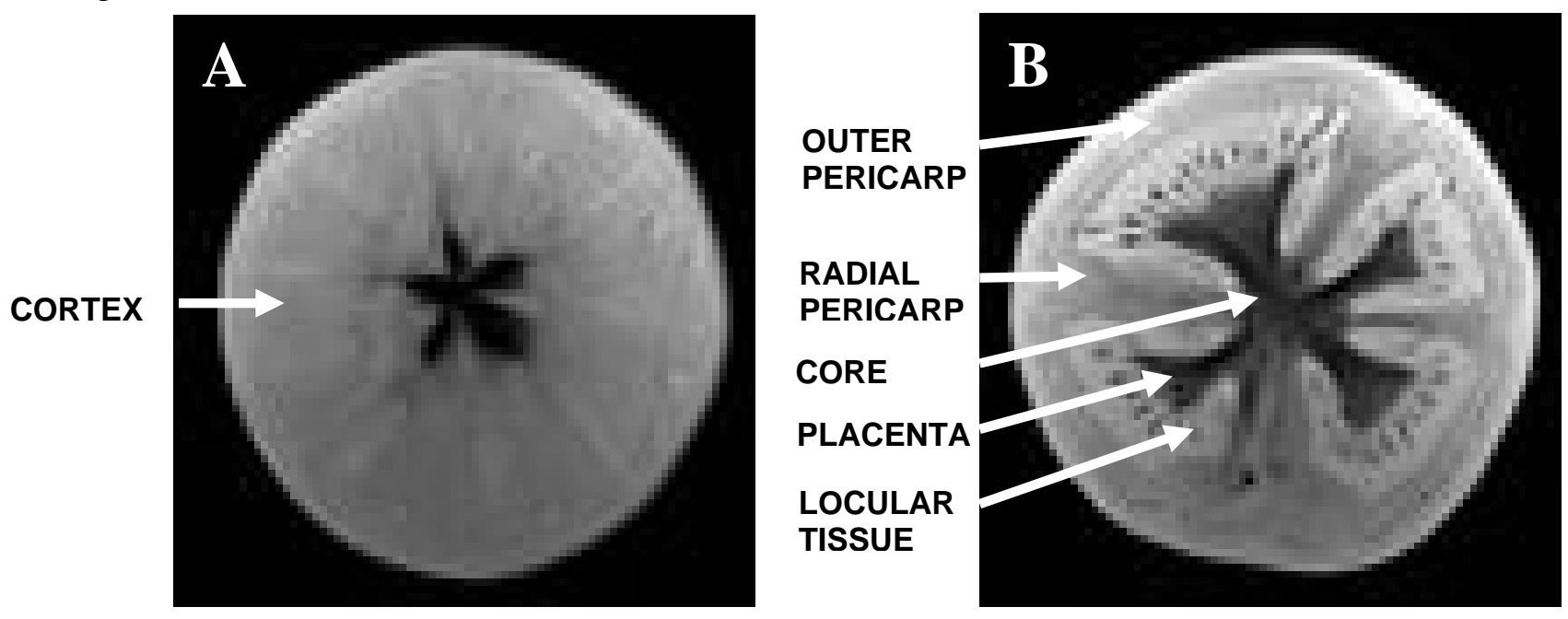
Fig. 2

A
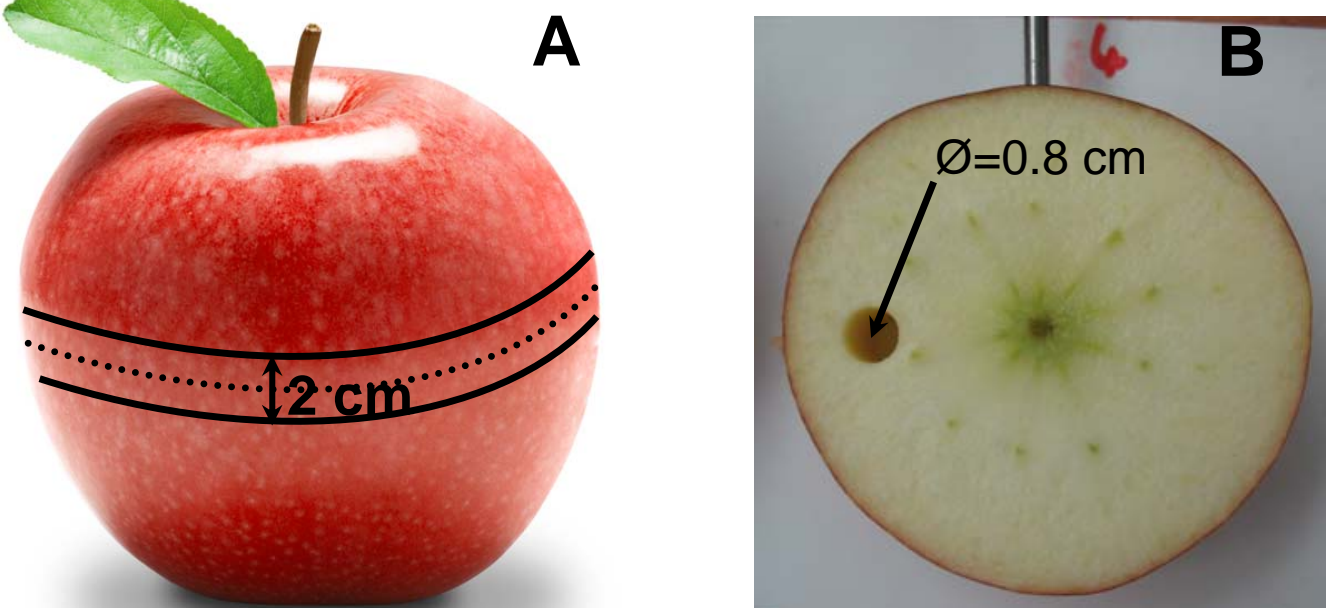

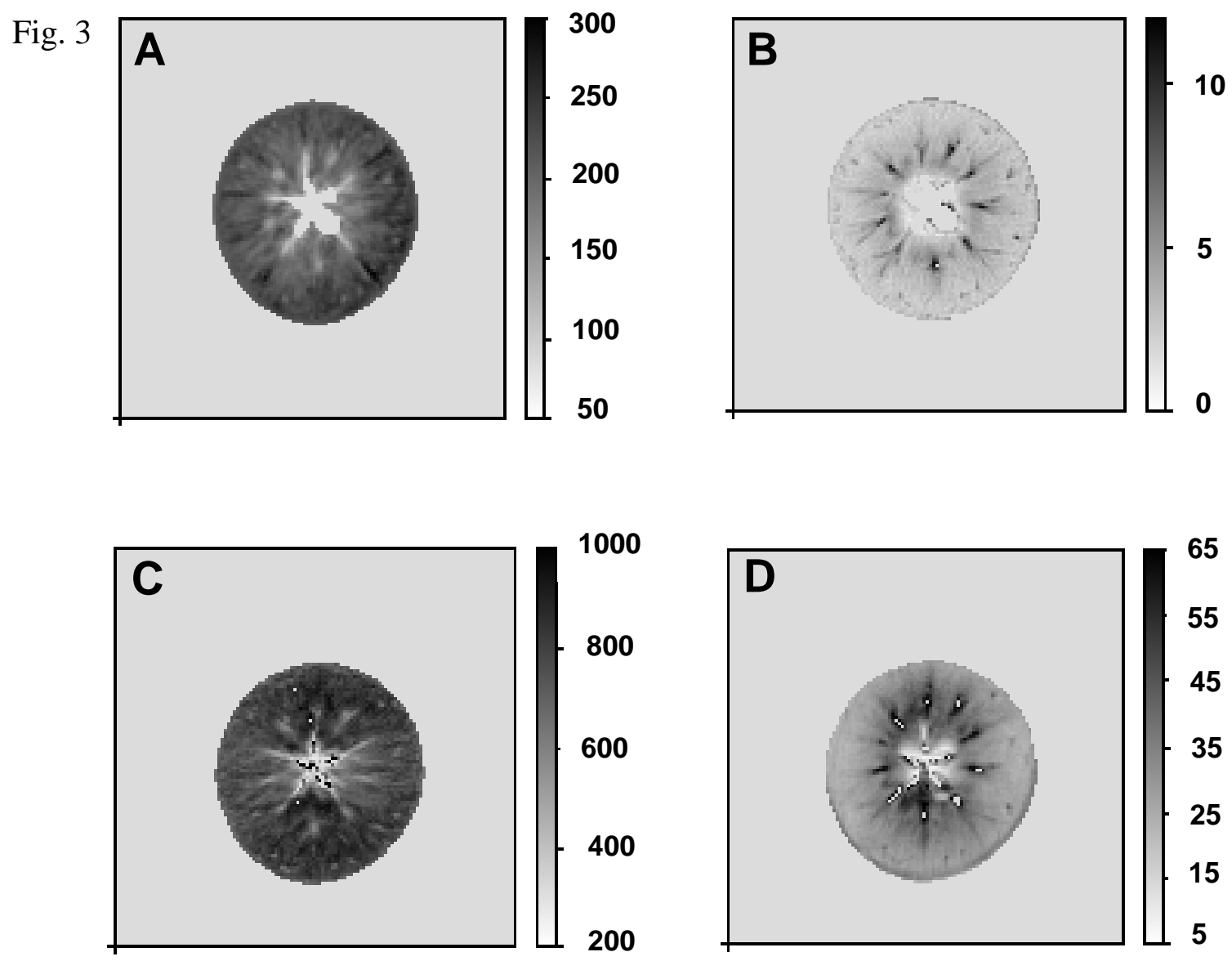
Fig. 4
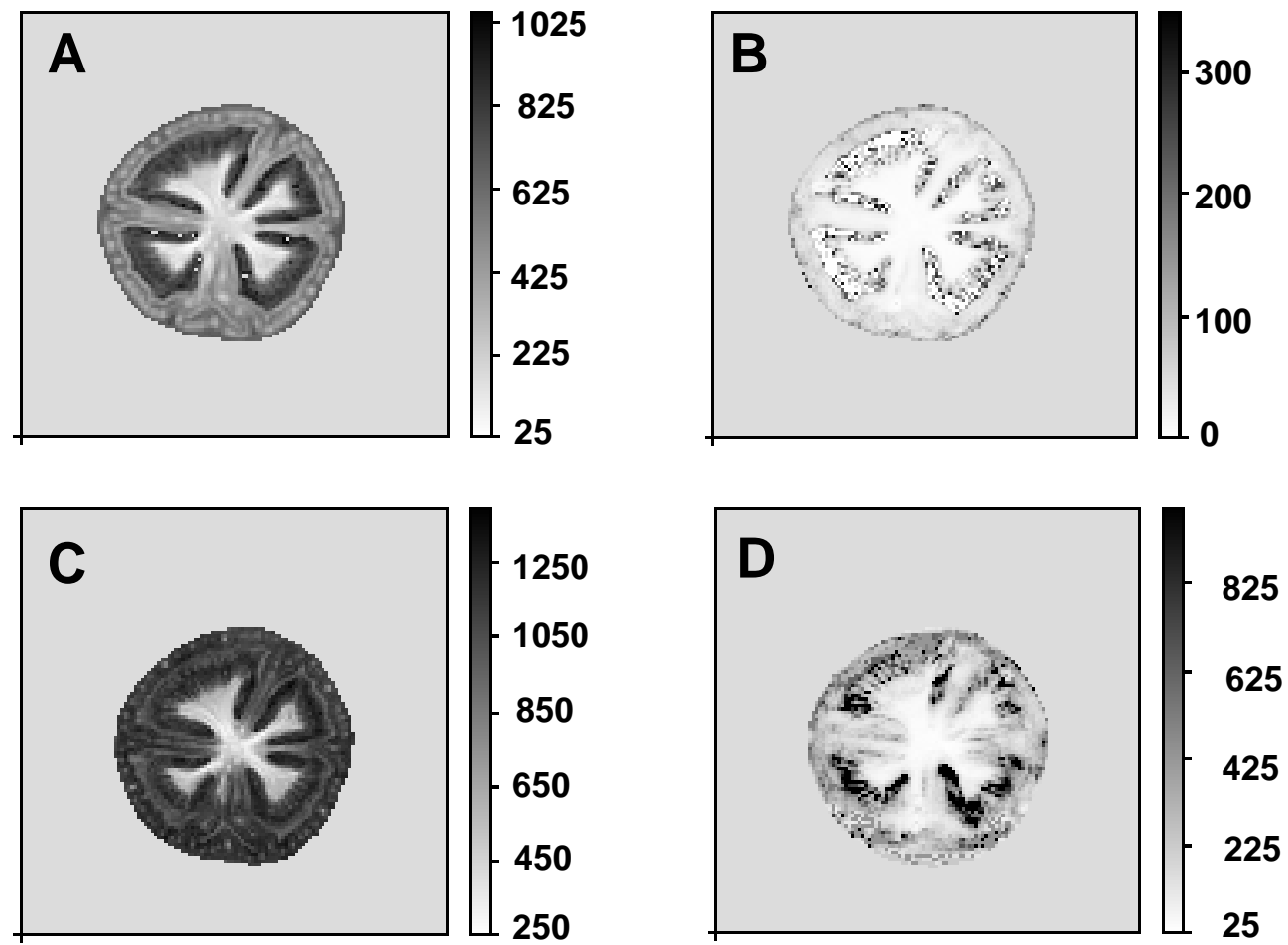

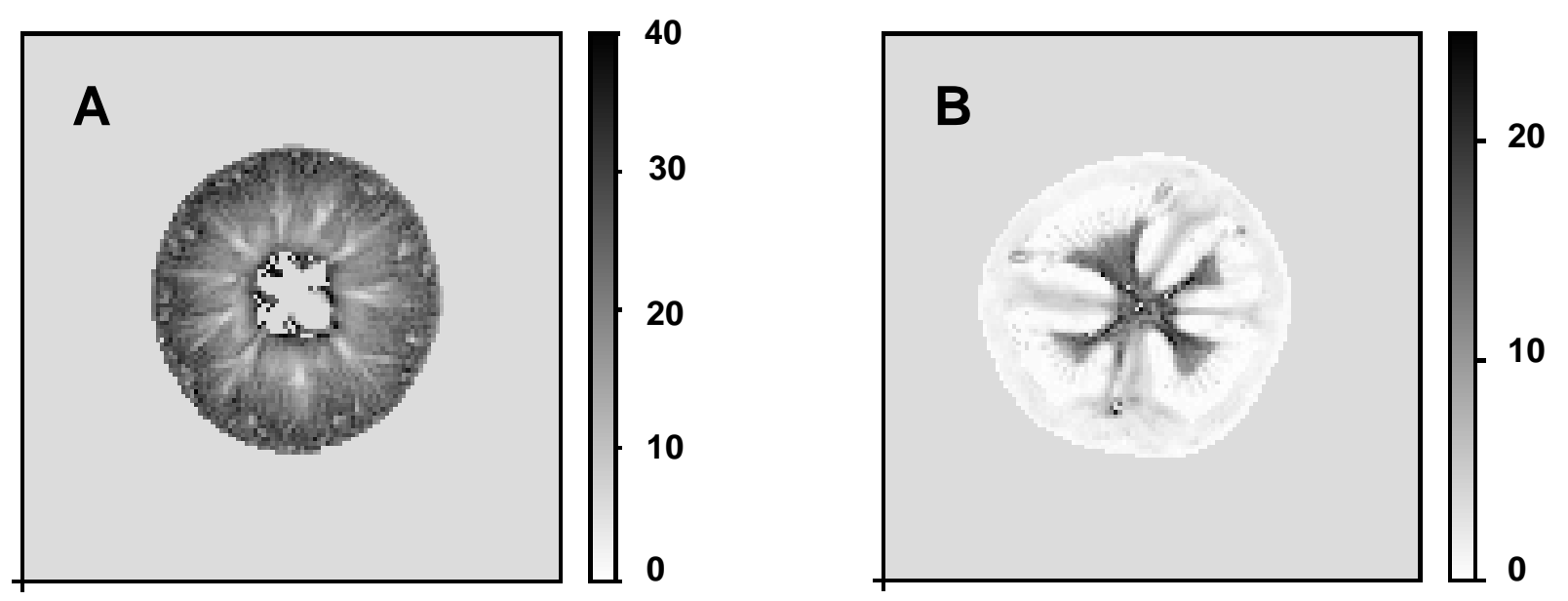

Fig. 5 


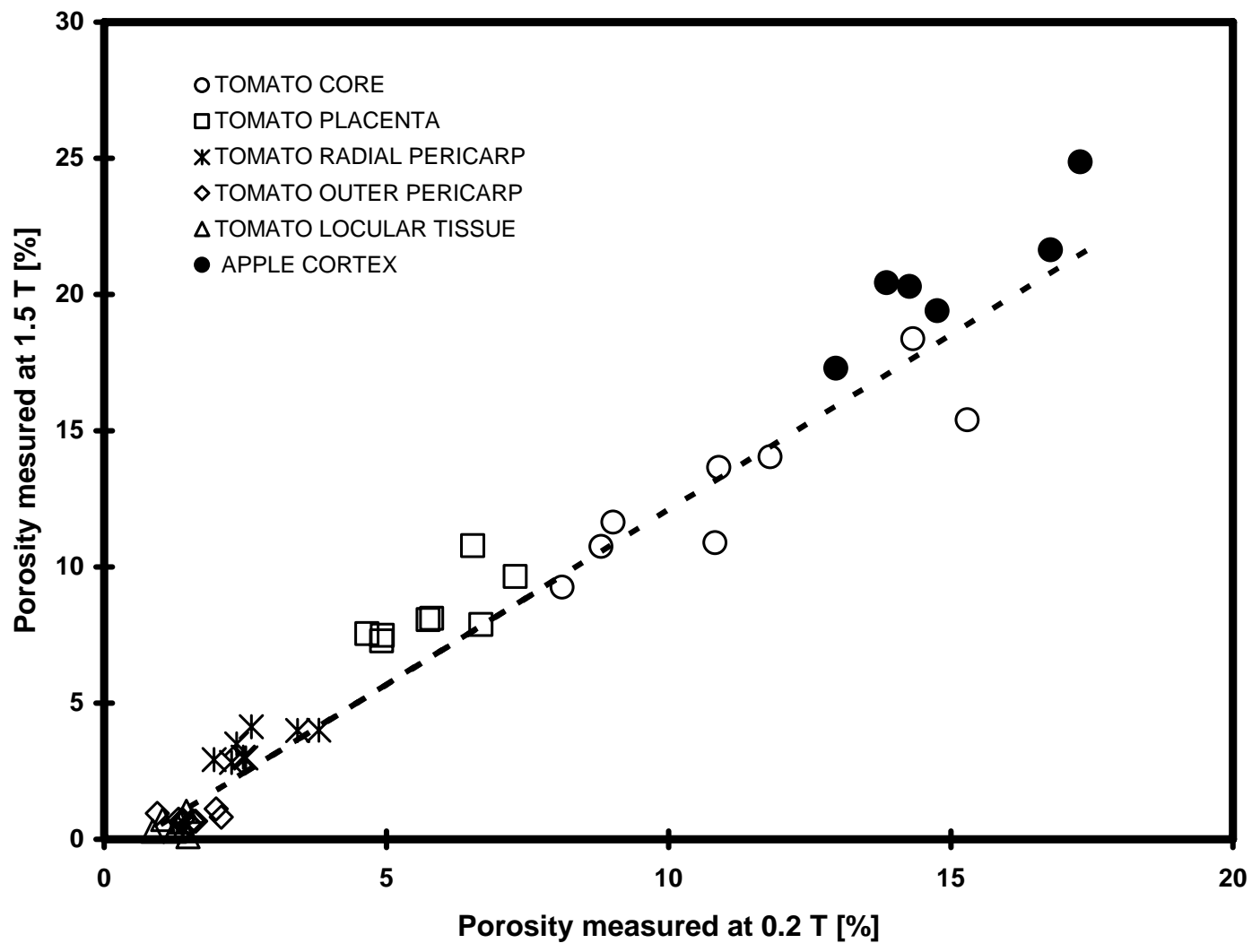

Fig. 6 
Fig. 7

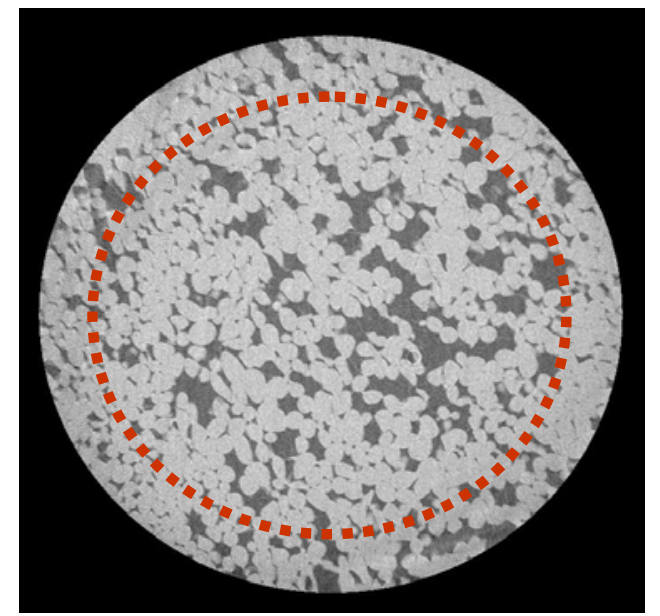


Fig. 8

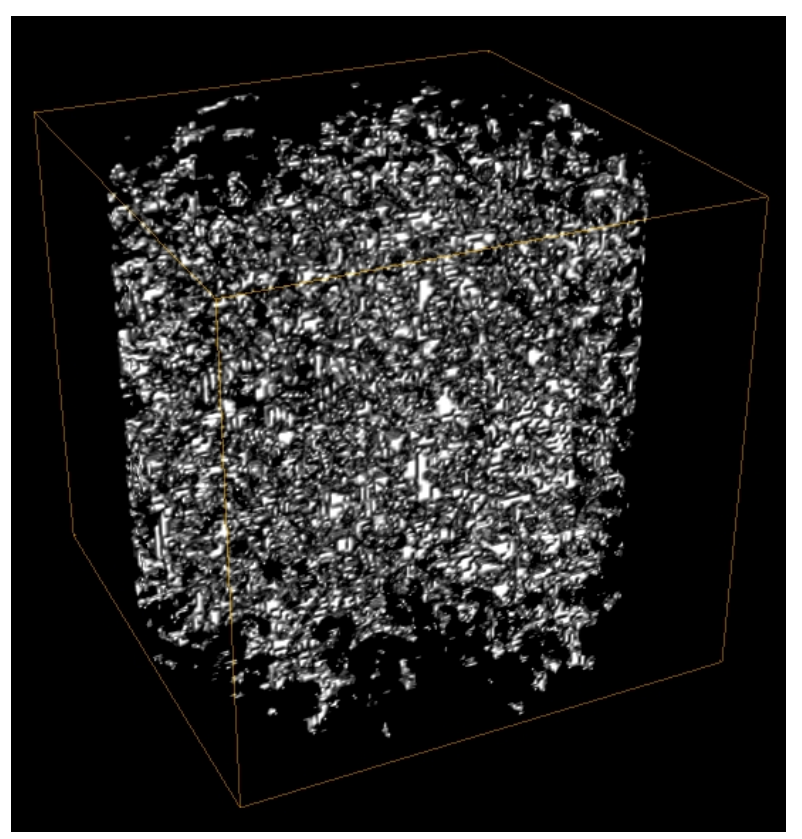




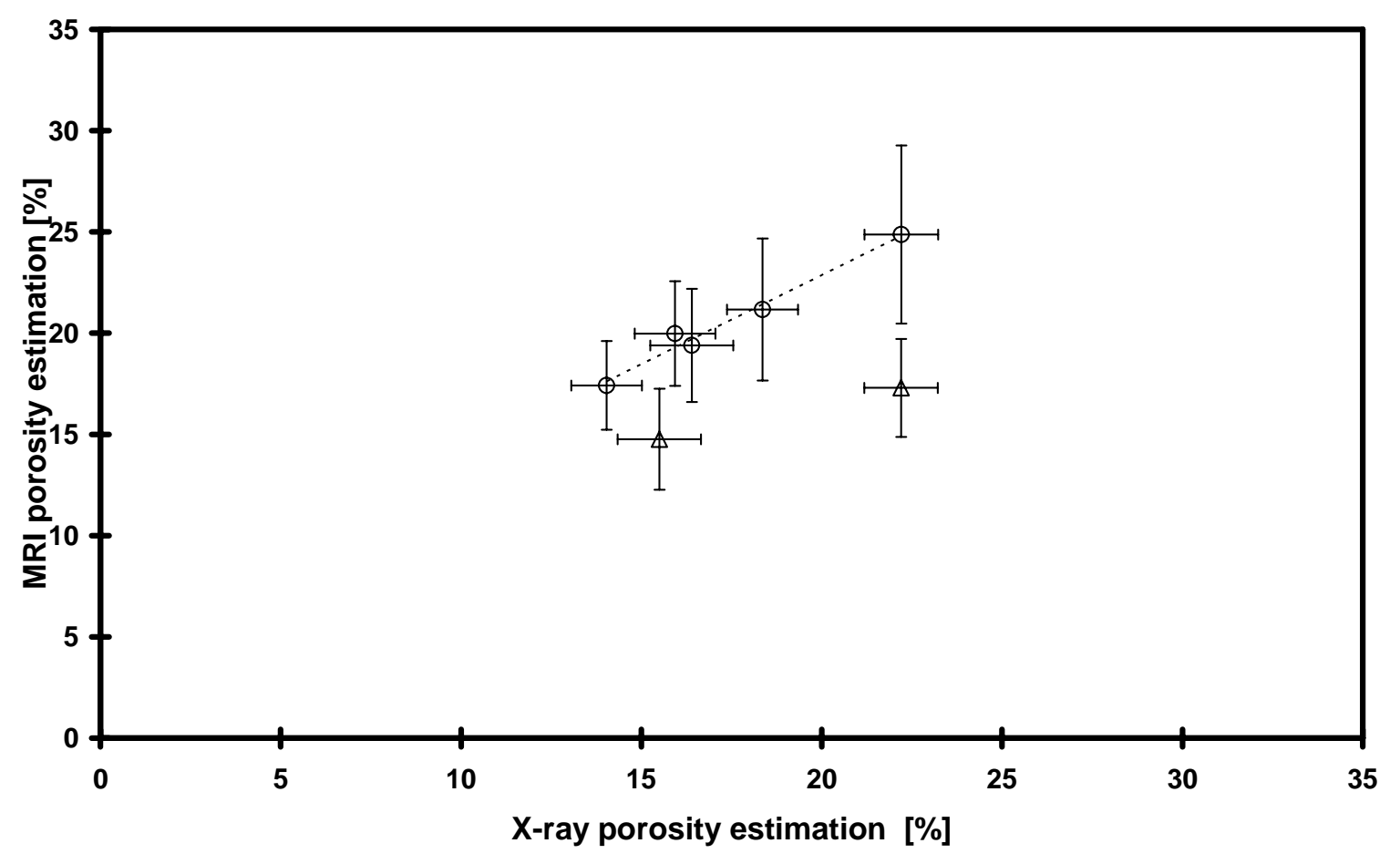

Fig. 9 
Tab. 1

\begin{tabular}{|l|l|rc|cc|}
\hline \multirow{2}{*}{ Fruit } & \multirow{2}{*}{ Tissue } & \multicolumn{2}{|c|}{$1.5 \mathrm{~T}$} & \multicolumn{2}{c|}{$0.2 \mathrm{~T}$} \\
\cline { 3 - 6 } & & $\mathrm{T}_{2}[\mathrm{~ms}]$ & $\mathrm{T}_{2}{ }^{*}[\mathrm{~ms}]$ & $\mathrm{T}_{2}[\mathrm{~ms}]$ & $\mathrm{T}_{2}{ }^{*}[\mathrm{~ms}]$ \\
\hline Apple & Cortex & $201 \pm 12$ & $3 \pm 0$ & $802 \pm 17$ & $34 \pm 4$ \\
\hline \multirow{4}{*}{ Tomato } & Core & $221 \pm 72$ & $5 \pm 1$ & $593 \pm 195$ & $45 \pm 10$ \\
& Placenta & $169 \pm 26$ & $8 \pm 1$ & $544 \pm 44$ & $78 \pm 12$ \\
& Radial pericarp & $416 \pm 35$ & $20 \pm 3$ & $995 \pm 53$ & $167 \pm 30$ \\
& Outer pericarp & $681 \pm 45$ & $79 \pm 14$ & $1137 \pm 49$ & $264 \pm 49$ \\
& Locular tissue & $817 \pm 67$ & $144 \pm 86$ & $1196 \pm 64$ & $308 \pm 45$ \\
\hline
\end{tabular}


Tab. 2

\begin{tabular}{|l|l|c|c|}
\hline \multirow{2}{*}{ Fruit } & \multirow{2}{*}{ Tissue } & \multicolumn{2}{|c|}{ Porosity [\%] } \\
\cline { 3 - 4 } & & $1.5 \mathrm{~T}$ & $0.2 \mathrm{~T}$ \\
\hline Apple & Cortex & $21 \pm 2$ & $15 \pm 2$ \\
\hline \multirow{4}{*}{ Tomato } & Core & $13 \pm 3$ & $11 \pm 3$ \\
& Placenta & $8 \pm 1$ & $6 \pm 1$ \\
& Radial pericarp & $3 \pm 1$ & $3 \pm 1$ \\
& Outer pericarp & $1 \pm 0$ & $2 \pm 0$ \\
& Locular tissue & $0 \pm 0$ & $1 \pm 0$ \\
\hline
\end{tabular}

